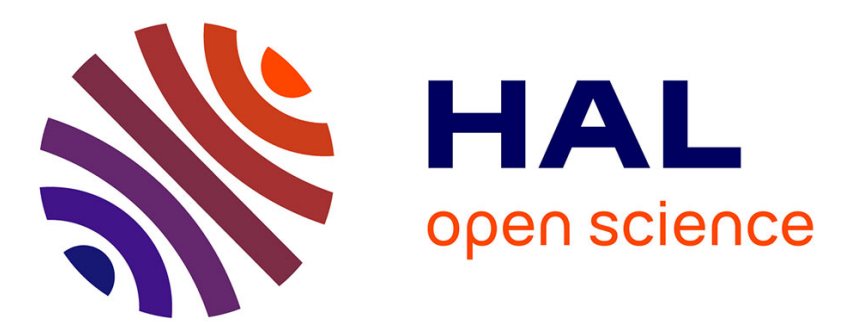

\title{
Phase imaging of supracellular structures with lens-free microscopy
}

\author{
Romain Alata, Thomas Vourc'H, Lionel Hervé, Sophie Morales, Pascal \\ Silberzan, Cédric Allier
}

\section{- To cite this version:}

Romain Alata, Thomas Vourc'H, Lionel Hervé, Sophie Morales, Pascal Silberzan, et al.. Phase imaging of supracellular structures with lens-free microscopy. Quantitative Phase Imaging VII, Mar 2021, Online Only, United States. pp.59, 10.1117/12.2579651 . hal-03367310

\section{HAL Id: hal-03367310 https://hal.science/hal-03367310}

Submitted on 11 Oct 2021

HAL is a multi-disciplinary open access archive for the deposit and dissemination of scientific research documents, whether they are published or not. The documents may come from teaching and research institutions in France or abroad, or from public or private research centers.
L'archive ouverte pluridisciplinaire HAL, est destinée au dépôt et à la diffusion de documents scientifiques de niveau recherche, publiés ou non, émanant des établissements d'enseignement et de recherche français ou étrangers, des laboratoires publics ou privés. 


\section{Phase imaging of supracellular structures}

with lens-free microscopy

Alata Romain ${ }^{1}$, Vourc'h Thomas ${ }^{2}$, Hervé Lionel ${ }^{1}$, Morales Sophie ${ }^{1}$, Silberzan Pascal ${ }^{2}$ and Allier Cédric

${ }^{1}$ CEA Leti-Grenoble ${ }^{2}$ Institut Curie-Paris

\section{INTRODUCTION}

Lens-free microscopes are simple setups, as they do not require any lens, but in return, they do not give directly an image of the sample but a hologram of it. Consequently, the final image must be reconstructed, but this reconstruction is often degraded because of the well-known phase wrapping phase. However, Convolutional Neural Networks (CNN) have been recently used to solve the phase wrapping problem in different field as digital holography. In this paper, we try this microscopy in order to reconstruct large and thick supracellular structures.

The objective is to allow phase imaging of supracellular structures posing the problem of wrapped phase with lens-free microscopy technique and deep learning based algorithm.

\section{METHODS}

Our method alternates between the deep learning and the inverse problem approach. We start by a first inversion giving a wrapped phase image and we use the CNN to unwrap it. Then we process a second reconstruction with the CNN Then we process a second reconstruction with the CNN
output as initialization to ensure that we fit well the data. This output as initialization to ensure that we fit well the data. This
algorithm architecture described in Fig. 1 is built like this in order to split different problems faced.

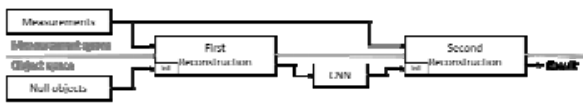

Fig. 1 : Algorithm architecture

In this paper, we focused on finding a good training dataset for a sample whose composition is evolving a lot with time. The sample is composed of fibroblasts and tumoral cells, and the later are duplicating while gatheng. This means that our reconstruction algorithm must be able to reconstruct the phase of small, thin and isolated objects, as well as big, thick and complex structures. That is why our dataset is composed of spherical cells (OPD ??), adherent cells (OPD ??), domes (OPD ??), and more complex structures as shown in Fig. 2.
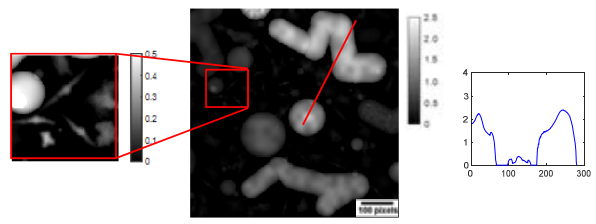

Fig. 2 : Part of one image of our training dataset with a magnification and a profile located by red square and line.

Our dataset is composed of every object encountered during the first 100 hours of the evolution of our samples.

\section{CNN CHARACTERISTICS}

Our network, described in Fig. 3, consist in a contracting and an expansive path giving him a shape of a ' $U$ ' and its name. The architecture of the network comes from [Unet biblio] where it is used for a segmentation task, but we see here that it is also efficient for a regression task. The training of this kind of networks is also faster than usual ones thanks to shortcuts in the architecture, and they give better results when there are large object in the field of view thanks to the maxpooling large

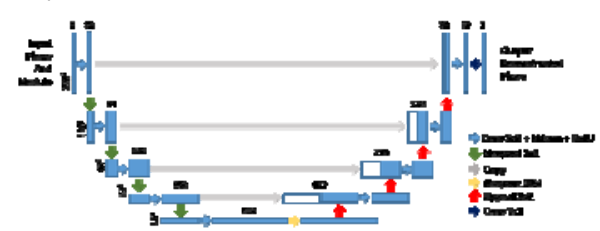

Fig. 3 : CNN architecture

\section{RESULTS}

Our algorithm reconstructs well isolated cells and medium structures with a diameter up to $300 \mu \mathrm{m}$ and an OPD as high as $2,5 \mu \mathrm{m}$, as Fig. 4 shows. For larger structures, the problem of wrapped phase reappears, as shown in Fig. 5. Phase breaks in several in the middle of those structures, as well as around them, and the reconstruction can't be trusted anymore. That said, isolated cells stay well reconstructed thanks to the second reconstruction of our algorithm as the magnification of the Fig 5 .

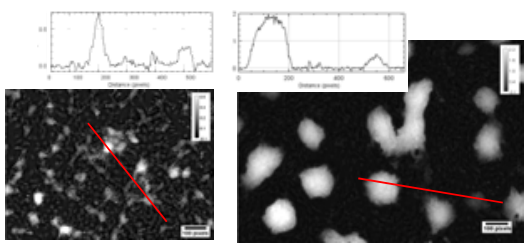

Fig. 4: Reconstruction of small and medium structures with profiles located by red lines

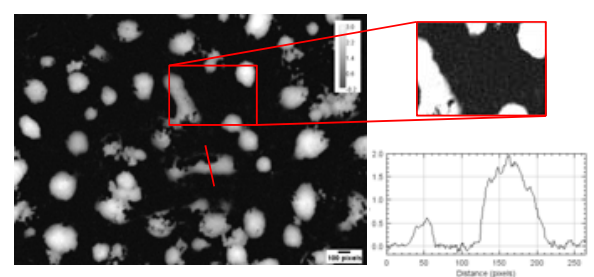

Fig. 5 : Reconstruction of large structures with a magnification and a profile located by red square and line

\section{DISCUSSION}

Adherent cells with an OPD lower than 0,5 $\mu \mathrm{m}$ are perfectly reconstructed as well as structures, up to $2,5 \mu \mathrm{m}$ and with a diameter of $300 \mu \mathrm{m}$

Our algorithm has shown good results for small object and is also able to reconstruct medium structures. Those results have been obtain with a single CNN which shows that it is possible to reconstruct more than $60 \mathrm{~h}$ of evolution of the sample without using a specific CNN for the different moment of the acquisition. Above that, it is hard to train the CNN for sall acquistion. Above that, it is hard to train the CNN for 作 adherent cells. Therefore, the concentration is high and phase variation are similar to what we observe at the top of structures. Also, as we tested our CNN on acquisition taken during the evolution of the sample, we cannot directly compare our results with other imaging techniques. We tried to do it with fixed sample composed of the same kind of sample but failed to find structures thick enough to totally demonstrate the efficiency of our algorithm for medium structures. Also, those samples were not as clean as expected and presented hose samples were not as clean as expected and presented our CNN has not been able to reconstruct those images and an adaptation of our dataset might be needed to do so.

\section{CONCLUSIONS}

Our algorithm, associated to our CNN architecture and its training dataset, have shown good potential to reconstruct isolated cells as well as medium structures in the same acquisition. This allowed to reconstruct acquisitions of an evolving sample during approximately $80 \mathrm{~h}$ with a single $\mathrm{CNN}$. That said, we still need to check the fidelity of those reconstructions for larger and thicker object.

1. Dataset and CNN architecture are well adapted to our problem.

2. Our algrorithm allows to reconstruct structures with OPD up to $2 \mu \mathrm{m}$ on acquisitions and more than $3,5 \mu \mathrm{m}$ on simulations.

3. More tests must be done to check the trueness of our algorithm for large and thick objects.

\section{REFERENCES}

1. Alternation of inverse problem approach and deep learning for lensfree microscopy image reconstruction, L. Hervé, DCA. Kraemer, O. Cioni, O. Mandula, M. Menneteau, S/ Morales, C Allier

2. End-to-end deep learning framework for digital holographic reconstruction, Z. Ren, Z. Xu, E.Y. Lam

4. One-step robust deep learning phase unwrapping, J. Zhang, X. Tian, J. Shao, H. Luo, R. Liang

3. U-Net : Convolutional Networks for Biomedical Image Segmentation, O. Ronneberger, P. Fischer, T. Brox 